\title{
INTERVENCIONES DE ENFERMERÍA EN TRAUMA CRANEOENCEFÁLICO EN URGENCIAS
}

\author{
Adriana Quiroga*, Johanna Ávila**, Giovanni Badillo**, Oswaldo Cleves**, Magda Garavito**, \\ Laura Huertas**, Landys Matallana** y Jenny Soto**
}

\section{Resumen}

Serie de 29 casos y sus resultados en observaciones realizadas con ayuda de una lista de chequeo durante el transcurso de los turnos diurnos y nocturnos de enfermería en el servicio de urgencias de un hospital de nivel III en un lapso de 30 días. Para recolectar la información se utilizó una lista con 54 ítems que describieron el manejo ideal del paciente con TCE, así como las intervenciones ejecutadas, las omitidas y los responsables de las mismas, para determinar cuáles deben realizarse durante los primeros 60 minutos del ingreso al servicio de urgencias. Según la literatura, el número de actividades que deben efectuarse son 54 , de las cuales 18 (33\%) son competencias del profesional de enfermería. Este estudio además reveló un factor importante como es el diagnóstico preciso del déficit neurológico y la regularidad de la valoración, lo que nos lleva a pensar que es indispensable realizar un protocolo de intervenciones de enfermería con pautas secuenciales para una mejor atención.

Palabras clave: trauma craneoencefálico, intervenciones en urgencias, lesión cerebral.

Abreviaturas: TCE, trauma craneoencefálico; APH, atención prehospitalaria.

\section{EMERGENCY ROOM NURSING INTERVENTIONS IN CRANEOENCEPHALIC TRAUMA}

\section{Abstract}

This study discusses a series of 29 cases and their results based on check-list assisted observations performed during ER nursing day and night shifts at a level III hospital in a 30-day period. A 54-item check-list which described the management of patients with CET, as well as implemented and omitted interventions, and person responsible, in order to determine which should be performed upon the first 60 minutes of patient admission to the ER, was used. According to literature, 54 interventions should be performed, 18 (33\%) of which must be carried out by the nursing professional. Additionally, this study reveals an important factor as is obtaining an accurate baseline and ongoing neurological deficit diagnosis which leads us to consider that a nursing intervention protocol including sequential guidelines in order to deliver better care is required.

Key words: craneoencephalic trauma, interventions at the ER, brain injury.

Fecha recibido: agosto 4 de 2008 - Fecha aceptado: mayo 6 de 2009

Asesora metodológica: Merideidy Plazas. Docente División de Investigaciones, Fundación Universitaria de Ciencias de la Salud. Bogotá DC. Colombia.
* Enfermera especialista en urgencias.

** Estudiantes VII semestre Facultad de Enfermería, Fundación Universitaria de Ciencias de la Salud, Bogotá DC. Colombia. 


\section{Introducción}

El TCE es una patología que existe desde el principio de la humanidad, aunque los diferentes tipos y mecanismos de lesión han cambiado en relación con el desarrollo tecnológico. El advenimiento de los vehículos de motor ha causado un incremento exponencial en nuestro siglo, lo cual nos ha motivado a buscar medidas de prevención, así como nuevas y mejores opciones terapéuticas en la atención de estos pacientes. Podemos decir que el TCE se considera una patología de la sociedad moderna.

Se produce por la acción de una fuerza mecánica sobre la cabeza que se transmite al cuero cabelludo, cráneo y masa encefálica. En los mecanismos se deben tener en cuenta la velocidad, aceleración, desaceleración y fuerza del impacto. Los tipos de lesión en un TCE son las primarias que suceden como resultado directo del traumatismo (ej. contusión, laceración, hematomas y hemorragias subaracnoideas) y secundarias las que aparecen después de las anteriores y que por lo regular están relacionadas con hipotensión e hipoxia. ${ }^{1}$

El estado clínico del enfermo con TCE es de suma importancia para el abordaje terapéutico, que se basa principalmente en la escala del coma de Glasgow asociada con el mecanismo de trauma y las lesiones que presenta al momento de su primera evaluación. El TCE es la lesión neurológica más frecuente; su incidencia aumenta en forma paulatina y causa la mitad de las muertes relacionadas con los traumatismos en personas jóvenes. ${ }^{2}$ Cerca del $80 \%$ de los pacientes con TCE que reciben atención médica pueden ser clasificados leves, $10 \%$ moderados y $10 \%$ severos. $^{2}$ En Estados Unidos cada año mueren 52.000 personas y 80.000 padecen discapacidad neurológica severa como consecuencia de trauma cerebral, que es la principal causa de muerte en el grupo de 18 a 44 años. ${ }^{3}$ En Colombia la tasa de mortalidad asociada con TCE de 300 muertes por cada 100.000 habitantes. ${ }^{3}$ Las causas más frecuentes de acuerdo con el DANE son: violencia interpersonal, suicidio, accidentes vehiculares y de otro tipo. En el año 2003 se registraron 5.492 muertes en accidentes de tránsito, que con respecto a las reportadas en el año 2002 (6.063) hay una disminución de $9,42 \%$ equivalente a 571 vidas salvadas. ${ }^{2}$ En cuanto a los grupos de edad, los lesionados o muertos oscilaron entre 18 y 45 años centrándose la mayoría de las víctimas (23\%) entre 25 y 34 años. $^{2}$ Con base en este porcentaje se identificó una alta incidencia de morbimortalidad en adultos jóvenes, motivo para basar este estudio en el anterior rango de edad.

Para determinar las intervenciones realizadas al paciente con TCE es indispensable conocer las estadísticas. Es desafortunado que en nuestro medio no disponemos de datos en cuanto a la frecuencia de lesiones craneoencefálicas en niños o adultos. El TCE es un problema mundial de salud pública. El número de casos por año en 100.000 habitantes en países industrializados oscila entre 100 y 250 casos, mientras una ciudad como Cali, presenta al año 600 de cada 100.000 habitantes, es decir una proporción 1:3.

El objetivo general del trabajo es determinar las intervenciones que se deben realizar en los pacientes con TCE para crear un protocolo de intervenciones de enfermería que mejore la atención al grupo de pacientes mencionados. La primera etapa fue determinar las intervenciones de enfermería que se realizan en los pacientes con TCE durante los primeros 60 minutos del ingreso al servicio de urgencias.

Los objetivos específicos propuestos fueron identificar las actividades que se aplican en la actualidad en el servicio de urgencias en la primera hora de atención, registrar las intervenciones realizadas por el equipo de salud a los pacientes con TCE, analizar los datos obtenidos en el instrumento de medición y mencionar conclusiones finales que sirvan de base para la discusión en los grupos focales y con la culminación de lo anterior poder crear el protocolo de intervenciones de enfermería en el paciente con TCE.

El equipo interdisciplinario desempeña un papel fundamental a nivel administrativo-asistencial, por ello es importante fusionar estos aspectos para brindar atención adecuada y tratamiento oportuno, reduciendo las complicaciones y mejorando el desenlace del lesionado. A lo largo de este estudio se investigó el TCE de forma observacional y analítica, y los comportamientos, patrones y actividades que realiza el profesional de enfermería, que de una u otra forma pueden llegar a ser definitivos para el paciente. Por tal razón, consideramos que la creación de un protocolo de intervenciones para el paciente con TCE aplicado a la realidad de los servicios de 
salud colombianos y a la atención directa que presta el personal de enfermería, mejorará la calidad en la atención prestada y así mismo el pronóstico de esta patología. Además, el grupo investigador pretende concebir en el lector un pensamiento crítico y creativo, en pro de generar la creación de nuevos protocolos, guías y procedimientos que permitan mejorar la atención de la población colombiana.

\section{Métodos}

Este trabajo que busca la creación de un protocolo de intervenciones de enfermería durante los primeros 60 minutos de atención al paciente con TCE se realizó en dos etapas; en la primera, el estudio diseñado fue una serie de casos, donde se observó durante los turnos diurnos y nocturnos las intervenciones que se le realizaron a 29 pacientes con TCE en el servicio de urgencias de un hospital de tercer nivel. La segunda fase fue el análisis estadístico para determinar resultados. La observación se realizó durante 30 días y se registró en un instrumento (lista de chequeo), conformado por 54 ítems propios de las actividades a realizar a un paciente con trauma severo, donde se encuentran contemplados: datos de ingreso, antecedentes, actuaciones iniciales, apertura de la vía aérea, buena ventilación, circulación, control de déficit neurológico y exposición del paciente. Estas detallan el manejo ideal del equipo de salud (médico, enfermero, auxiliar) en casos de TCE y se construyó basado en la guía de manejo de la Advanced Trauma Life Support ${ }^{4}$ y las de manejo del Ministerio de Protección Social. ${ }^{5}$ Los objetos de estudio fueron 29 pacientes que ingresaron al servicio de urgencias. El tipo de muestreo fue no probabilístico.

Los investigadores ingresaron al servicio de urgencias y se ubicaron en el área de reanimación, registrando en la lista de chequeo las intervenciones efectuadas durante los primeros 60 minutos de ingreso del paciente con TCE. Para controlar el sesgo se realizó una capacitación previa al equipo de observadores, se estandarizó por pares y se consignaron las intervenciones omitidas, ejecutadas y el responsable de las mismas. Sin embargo, la información recolectada sólo fue una guía para iniciar los grupos focales, lo cual redujo de manera sustancial las posibilidades de un sesgo. Una vez terminadas las listas de chequeo, se procesó la información y se sometió al análisis estadístico descriptivo para obtener los resultados finales de la investigación. Para tal efecto, se utilizó el paquete estadístico Microsoft Office Excel 2003.

\section{Resultados}

Durante el periodo de estudio se observaron 29 personas entre 25 y 35 años que consultaron al servicio de urgencias de un hospital de tercer nivel de atención por presentar TCE. La gravedad, medida por la escala del coma de Glasgow, fue leve en 26 casos (90\%) y severa en el restante; 19 pacientes $(66 \%)$ recibieron atención prehospitalaria y el porcentaje restante fue trasladado por otros medios (servicio particular, público y patrulla); dos $(8 \%)$ presentaron antecedentes de patologías (uno hipertensión arterial y otro desórdenes de la coagulación).

El tipo de lesión que se presentó en todos los observados fue mecánica, donde el 59\% ocurrió por accidente automovilístico (17 pacientes); cinco tenían lesión contusa (17\%); cuatro por caídas (14\%), uno por arma de fuego $(3,4 \%)$, uno por arma blanca $(3,4 \%)$ y uno de causa desconocida (3,4\%); 18 pacientes (62\%) se encontraban bajo efectos de sustancias psicoactivas: alcohol en 16 y en dos marihuana y escopolamina.

En el TCE leve se observó que la alineación de columna y colocación del collar cervical fue realizada en un alto porcentaje por el personal de APH (Tabla 1) Durante la APH a tres pacientes (100\%) con TCE severo se les realizó la alineación e inmovilización de la columna cervical y se trasladaron en bloque. Las intervenciones en la apertura de la vía aérea no fueron necesarias en la mayoría con TCE leve (Tabla 2). Se ejecutó subluxación mandibular y observación de la cavidad oral en busca de objetos extraños por personal de APH. En dos de los pacientes con TCE severo se realizó succión de la vía aérea o extracción de cuerpos extraños.

Buena ventilación: en la mayoría de los casos de TCE leve no se administró oxígeno durante los primeros 60 minutos de ingreso del paciente (Figura 1). El examen físico no se realizó a todos los pacientes con TCE leve a pesar de ser una actividad primordial durante la atención (Figura 2). Se practicó por el médico en el 67\% con TCE severo, ejecutando las siguientes maniobras: inspección, palpación y auscultación toráxica. 


\begin{tabular}{|l|c|c|c|c|c|}
\hline \multicolumn{5}{|c|}{ Tabla I. Actuaciones iniciales en TCE leve } \\
\hline Intervención inicial & $\begin{array}{c}\text { No realizados } \\
\%\end{array}$ & $\begin{array}{c}\text { Médico } \\
\%\end{array}$ & $\begin{array}{c}\text { Enfermero } \\
\%\end{array}$ & $\begin{array}{c}\text { Auxiliar } \\
\%\end{array}$ & $\begin{array}{c}\text { APH } \\
\%\end{array}$ \\
\hline Alineación columna cervical & 38 & 0 & 0 & 0 & 62 \\
Collar cervical & 38 & 0 & 0 & 0 & 62 \\
Traslado en bloque & 58 & 10 & 0 & 0 & 42 \\
\hline
\end{tabular}

\begin{tabular}{|l|c|c|c|c|c|}
\hline \multicolumn{5}{|c|}{ Tabla 2. Apertura de la vía aérea en TCE leve } \\
\hline Apertura de la vía aérea & $\begin{array}{c}\text { No realizados } \\
\%\end{array}$ & $\begin{array}{c}\text { Médico } \\
\%\end{array}$ & $\begin{array}{c}\text { Enfermero } \\
\%\end{array}$ & $\begin{array}{c}\text { Auxiliar } \\
\%\end{array}$ & $\begin{array}{c}\text { APH } \\
\%\end{array}$ \\
\hline Subluxación mandibular & 88 & 0 & 0 & 0 & 12 \\
Objetos extraños & 88 & 12 & 0 & 0 & 0 \\
Succión vía aérea & 100 & 0 & 0 & 0 & 0 \\
\hline
\end{tabular}

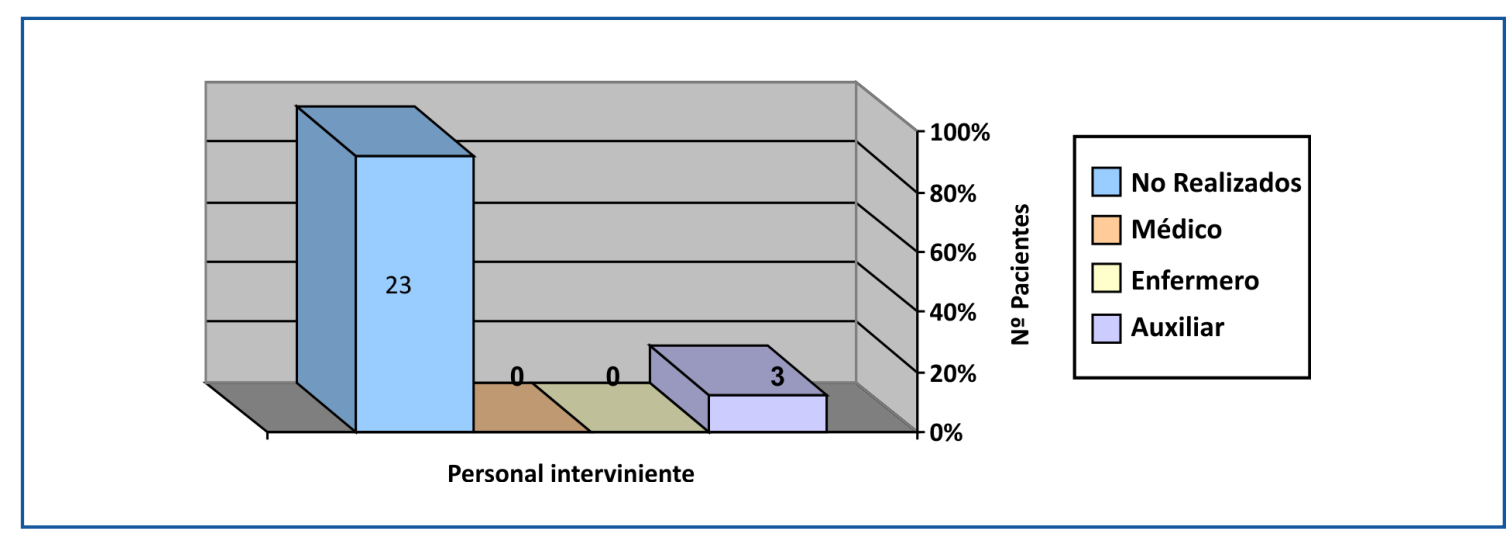

Figura I. Administración de oxígeno a pacientes con TCE leve.

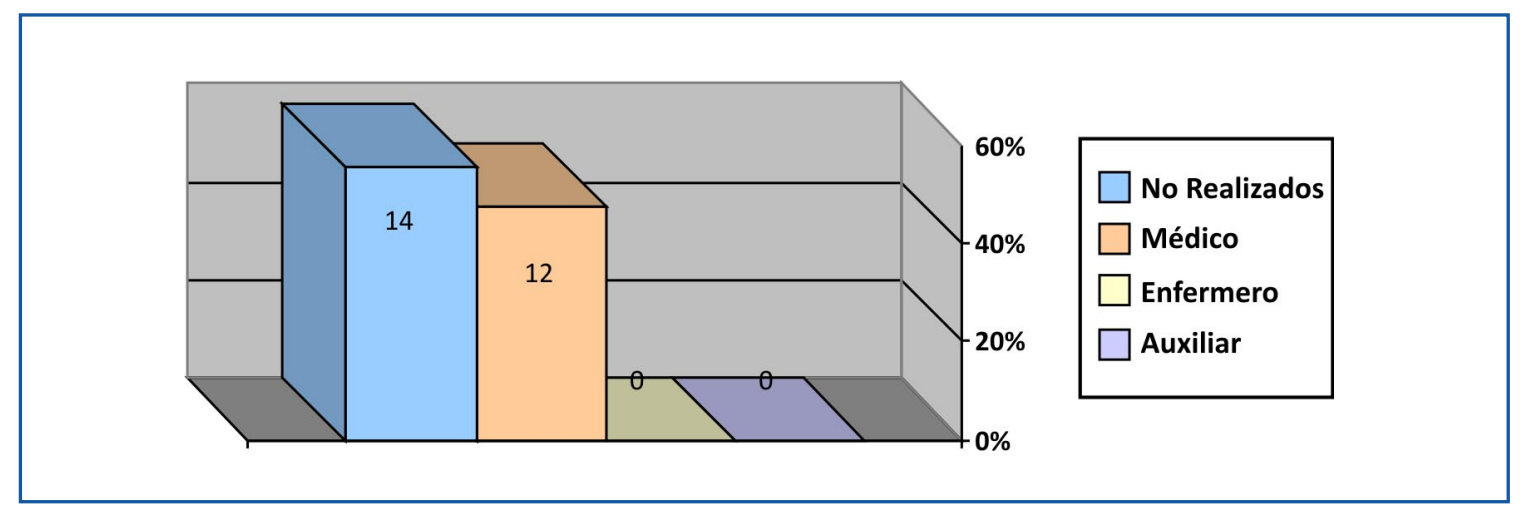

Figura 2. Examen físico al paciente con TCE leve.

Circulación: el médico verificó presencia de hemorragias en algunos de los pacientes con TCE leve (Figura 3). De los 8 observados, cuatro fueron controlados por el equipo médico (Figura 4). En el 100\% de los pacientes con TCE severo se verificó la presencia de hemorragias, de las cuales el $66 \%$ fueron controladas por el médico aplicando presión directa sobre la herida. 

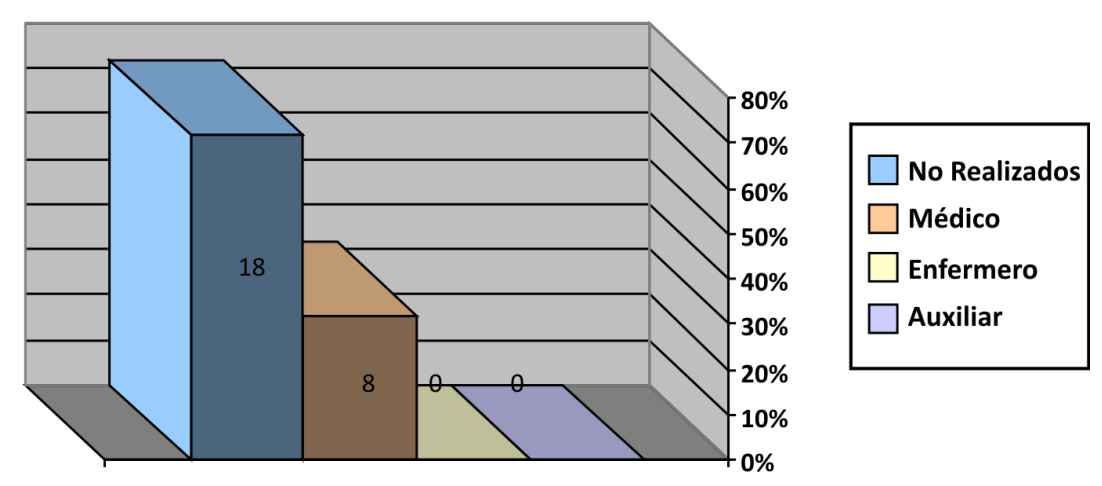

Figura 3. Verificación de hemorragias en TCE leve.

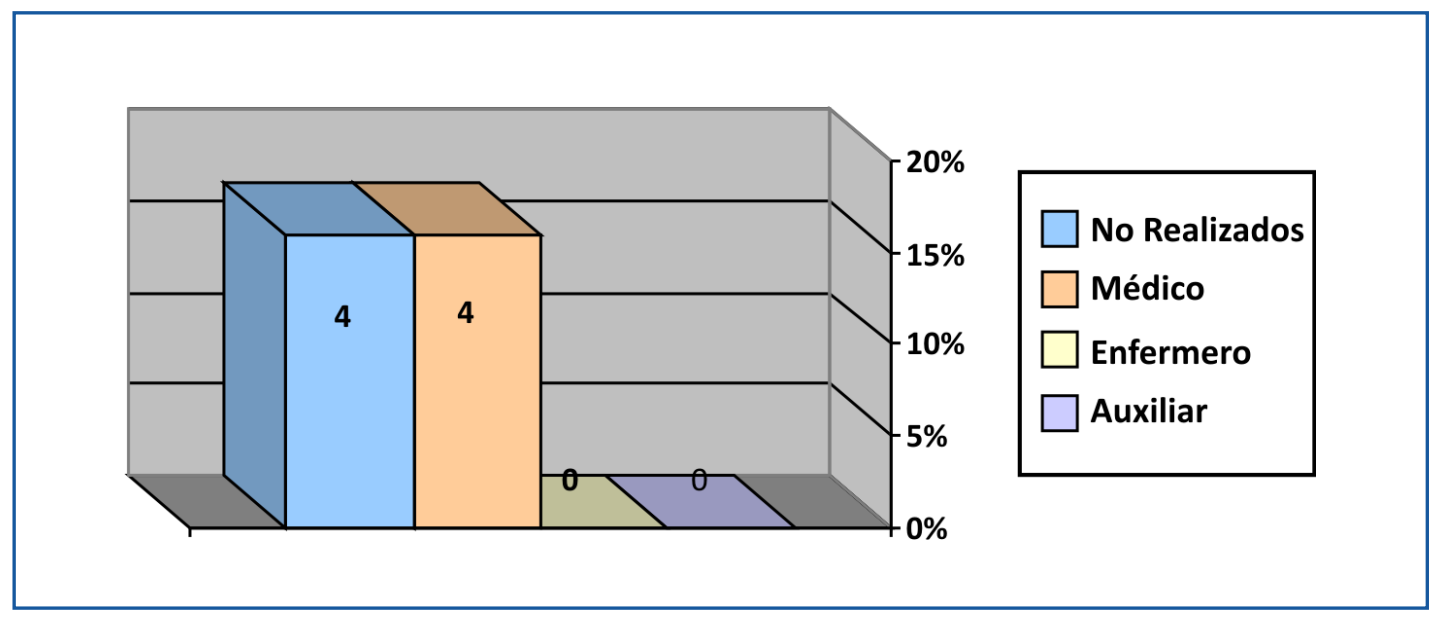

Figura 4. Control de hemorragias con TCE leve.

La administración de líquidos, se realizó en casi la mitad de los pacientes con TCE leve y fue efectuada en su mayoría por enfermero (Figura 5). Se efectuó reanimación con líquidos endovenosos en todos los que tenían TCE severo, utilizando soluciones cristaloides. La monitorización de los signos vitales es una intervención del equipo de salud; en el estudio, se detectó un bajo índice de esta actividad en casos de TCE leve (Figura 6). Ante TCE severo todos fueron monitorizados, en $66 \%$ por enfermero y $34 \%$ por auxiliar. La administración de medicamentos es una actividad propia del profesional de enfermería y lo fue en la mayoría con TCE leve (Figura 7) y en $66 \%$ con TCE severo.

El control neurológico es interdisciplinario y todo miembro del equipo de salud está en capacidad de efectuarlo.
Se destaca que durante los primeros 60 minutos de atención esta actividad no fue ejecutada de manera correcta en los pacientes con TCE leve, ya que no hubo secuencia con la valoración (Tabla 3). La valoración de la escala del coma Glasgow en paciente con TCE severo fue realizada por el médico con los siguientes intervalos: cinco minutos $66 \%$, diez minutos $34 \%$ y 30 minutos $34 \%$; en relación con la valoración pupilar ésta se llevó a cabo por el médico con los siguientes intervalos: cinco minutos $66 \%$ y diez minutos $34 \%$.

Durante el manejo del TCE es fundamental mantener la adecuada oxigenación cerebral para evitar posibles complicaciones, pues según la guía para el manejo hospitalario de urgencias ${ }^{5}$ el profesional de enfermería tiene el conocimiento científico acerca del manejo de dispositi- 


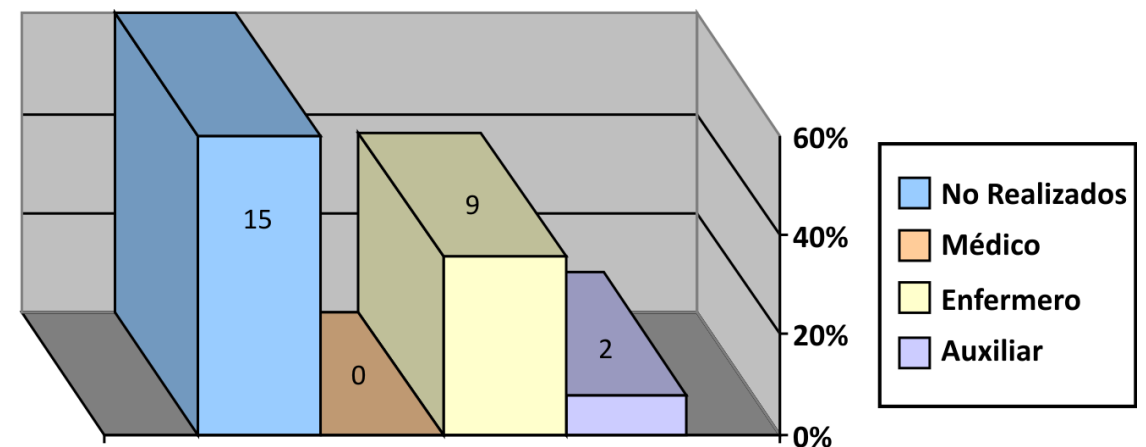

Figura 5. Administración de líquidos en pacientes con TCE leve.

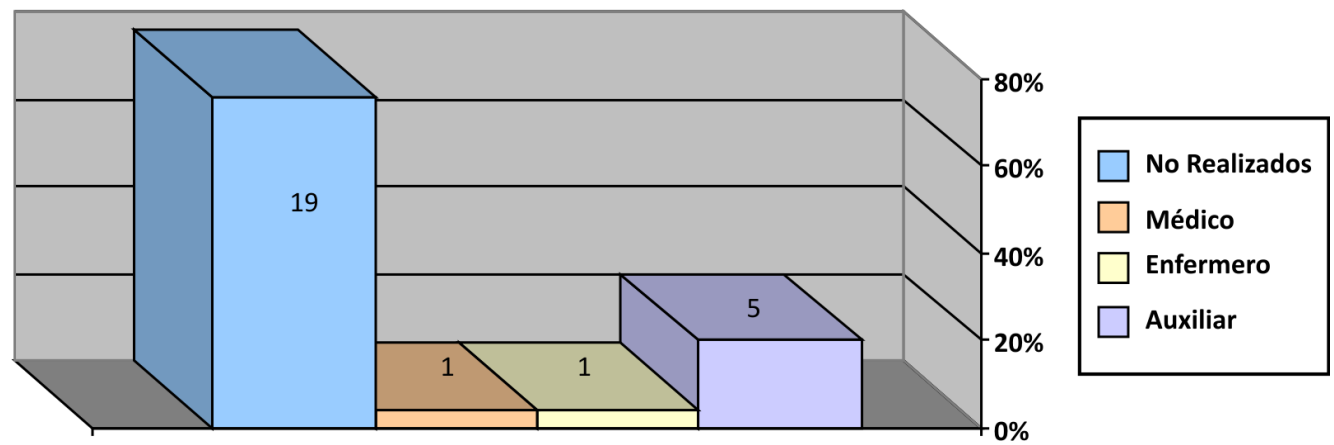

Figura 6. Monitorización del paciente con TCE leve.

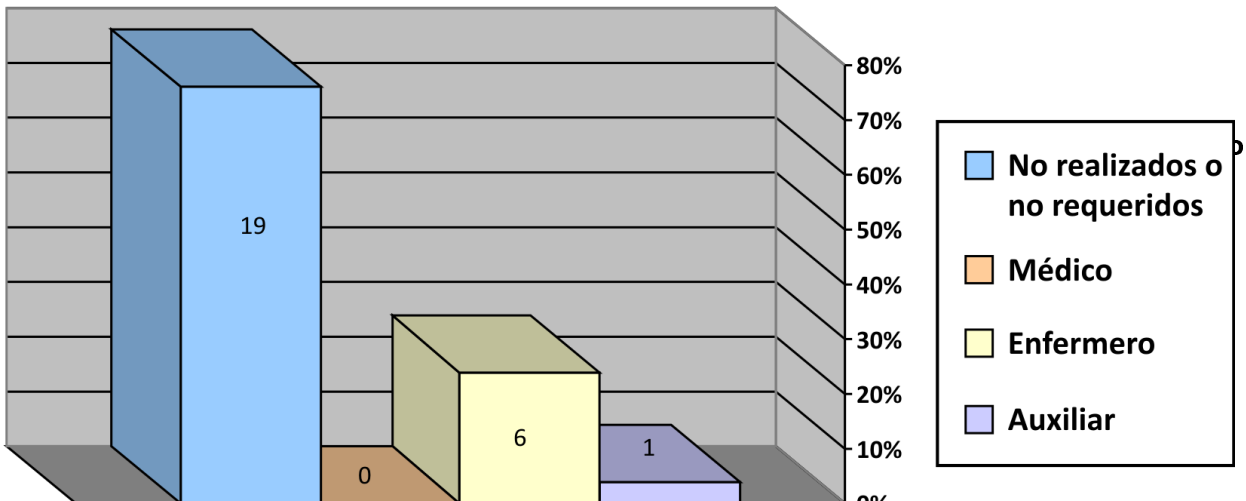

Figura 7. Administración de medicamentos en pacientes con TCE leve. 


\begin{tabular}{|l|c|c|c|c|c|}
\hline \multicolumn{5}{|c|}{ Tabla 3. Control neurológico del paciente con TCE leve } \\
\hline Intervención control neurológico & $\begin{array}{c}\text { No se realizó } \\
\%\end{array}$ & $\begin{array}{c}\text { Médico } \\
\%\end{array}$ & $\begin{array}{c}\text { Enfermero } \\
\%\end{array}$ & $\begin{array}{c}\text { Auxiliar } \\
\%\end{array}$ & $\begin{array}{c}\text { APH } \\
\%\end{array}$ \\
\hline Escala coma Glasgow $60 \mathrm{~min}$ & 100 & 0 & 0 & 0 \\
Escala coma Glasgow 30 min & 96 & 0 & 0 & 4 \\
Escala coma Glasgow 10 min & 92 & 4 & 0 & 0 \\
Escala coma Glasgow 5 min & 88 & 0 & 0 & 0 \\
Valora pupilas 60 min & 100 & 0 & 0 & 0 \\
Valora pupilas 30 min & 88 & 12 & 0 & 0 \\
Valora pupilas 10 min & 88 & 12 & 0 & 0 \\
Valora pupilas 5 min & 7 & 23 & 0 & 0 \\
\hline
\end{tabular}

vos, requerimientos y volúmenes ventilatorios de acuerdo con la condición clínica del paciente. Sin embargo, se detectó que el oxígeno es proporcionado por el personal auxiliar. Por otro lado, se observó que en un alto índice no se administró oxigenoterapia, siendo uno de los requerimientos principales en todo paciente con TCE.

El examen físico es un componente importante durante la valoración inicial del TCE. El Dr. A. Hernández $z^{6}$ afirma que el pronóstico en TCE leve o severo no es seguro cuando sólo se establece sobre la puntuación de la escala de Glasgow, siendo relevante el aporte del examen físico completo para verificar hallazgos que permitan evaluar la condición real y determinar tratamiento y pronóstico. Esto contradice los resultados del estudio, que indicaron que el examen físico no fue aplicado en la totalidad.

El control de las hemorragias se logra, según la gravedad de la lesión, con medidas de presión en la hemorragia externa o bien mediante hemostasia quirúrgica en la interna. Según el Colegio Americano de Cirugía en Advanced Trauma Life Support ${ }^{4}$, el profesional evalúa el llenado capilar, la coloración de la piel y la temperatura corporal para el manejo de hemorragias externas aplicando presión directa sobre la herida. En relación con el estudio, se vio que en algunos de los pacientes en los que se valoró la presencia de hemorragia, no se controló debido a que se trataba de lesiones que no representaban riesgo para la vida.

Se evidenció que la reanimación con líquidos en la mayoría de los casos no se realizó según las guías de mane- jo prehospitalario de trauma, procedimiento vital para el funcionamiento cardiovascular, prevenir lesiones cerebrales secundarias y evitar posibles complicaciones (choque, deshidratación, hipotensión o desequilibrio hidroelectrolítico). En la presente investigación esta intervención fue realizada en un alto porcentaje por el auxiliar de enfermería. Recordamos que según la guía para el manejo hospitalario de urgencias ${ }^{5}$ éste debe realizarlo personal con buen nivel de capacitación y destreza.

En el servicio de urgencias se observó que la administración de medicamentos no se realizó en la mayoría de pacientes, por no estar indicada en el momento de la atención; cuando lo fue, lo hizo casi siempre el profesional de enfermería, en concordancia con la literatura del manejo hospitalario de urgencias ${ }^{5}$ que cita: "La enfermera es la responsable de la correcta administración de medicamentos, la notificación, la valoración de sus efectos farmacocinéticos y farmacodinámicos, y según la terapéutica decidir la vía de administración”.

Los signos vitales constituyen una herramienta valiosa como indicadores del estado funcional del enfermo. Su registro no debe convertirse en una actividad automática o rutinaria; los resultados deben ser el reflejo de la evaluación clínica confiable. La interpretación adecuada y oportuna ayuda al enfermero y al médico a decidir conductas de manejo. Sin embargo, el estudio demostró que no fue efectuado en todos los pacientes, dejando de lado el indicador más importante para la valoración inicial del paciente con TCE. Tan pronto como el estado cardiopulmonar del paciente ha sido estabilizado, se procede a realizar la valoración neurológica rápida y dirigi- 
da. Consiste en determinar la escala coma de Glasgow y la respuesta pupilar. Estos dos indicadores son significativos y confiables sobre la severidad de la lesión encefálica, con mediciones repetidas para determinar mejoría o deterioro del estado. En relación con lo anterior, el estudio evidenció que a pesar de su importancia, el control de déficit neurológico no se llevó a cabo en todos los pacientes ni con la regularidad requerida.

Durante la primera fase de este estudio, se presentaron múltiples limitaciones como la carencia de tiempo por parte de los asesores, del equipo investigador y las directivas de los centros donde se efectuó el muestreo, así como la falta de accesibilidad por parte de la institución y la asimetría de la información.

\section{Recomendaciones}

- Realizar una mejor planeación y organización de las actividades investigativas.

- Elaborar un cronograma de actividades y ejecutarlo en forma estricta.
- Definir bien las competencias.

- Limitar el número de investigadores según la necesidad del proyecto.

\section{Referencias}

1. Stinson K, Sturt P, editores. Urgencias en enfermería. Barcelona: Océano; 2002.

2. Colombia. Instituto Nacional de Medicina Legal y Ciencias Forenses. Estadísticas Enero - Diciembre año 2003. Bogotá: INMLCF; 2003. $320 \mathrm{p}$.

3. Fundación de Trauma Craneoencefálico. Guías para el manejo prehospitalario del trauma craneoencefálico. Nueva York: BTF; 2000 .

4. Advanced trauma life support: guías para manejo de trauma. New Orleáns: ATLS, 1999. 195 p.

5. Colombia. Ministerio de la Protección Social: guías de manejo de urgencias. Bogotá: El Ministerio, 2000. 796 p.

6. Hernández A. Trauma craneoncefálico [monografía en internet]. Boston: National Academy Neurology; 2003 [citado 3 Jun 2009]. Disponible en: http:/www.neuroc.sld.cu 